\title{
Das Very early HELLP-Syndrom
}

Jan Pauluschke-Fröhlich

\begin{abstract}
Das „Very early HELLP-Syndrom“ ist eine schwerwiegende Schwangerschaftskomplikation. Die fürs HELLP-Syndrom typische Trias aus Hämolyse, ansteigenden Leberwerten und einer Thrombozytopenie tritt zu einem Zeitpunkt auf, an dem das Kind außerhalb des Mutterleibes noch nicht lebensfähig ist. Mütterliche Risiken sprechen dafür, die Schwangerschaft zu beenden. Doch betroffene Paare wollen ihr Kind in ein Gestationsalter bringen, an dem es mit Maximalversorgung extrauterin lebensfähig wäre. Gefragt sind fachliches Augenmaß, hohe menschliche Kompetenz und eine interdisziplinäre Strategie, um tragfähige Entscheidungen treffen zu können.
\end{abstract}

\section{Hintergrund}

Beim Very early HELLP-Syndrom sind es vor allem die mütterlichen Risiken, die zu einer raschen Beendigung der Schwangerschaft drängen. Dem steht der erklärte Wunsch betroffener Paare gegenüber, den Fetus-unter Ausschöpfung aller medizinischen Möglichkeiten - in ein Gestationsalter zu begleiten, zu dem eine Maximalversorgung außerhalb des Mutterleibes möglich wird. Dabei darf nicht allein auf die Grenze der extrauterinen Lebensfähigkeit geschaut werden, sondern es muss zukunftsweisend abgewogen werden, unter welchen Voraussetzungen der Neonatologe eine Maximaltherapie bzw. Unterstützung des Kindes postpartal beginnen soll.

Das von Weinstein im Jahr 1982 erstmals beschriebene HELLP-Syndrom stellt eine schwerwiegende Schwangerschaftskomplikation dar [1]. Sie tritt bei 0,5-0,9\% aller Schwangerschaften auf. Je nach Gestationsalter bei Geburt ist diese Erkrankung mit einer für die betroffenen Frauen und Kinder 1,1\%-igen maternalen und einer 7,4-34\%-igen fetalen Mortalität verbunden.

\section{Symptome und Therapieoptionen des HELLP-Syndroms}

Charakterisiert wird das HELLP-Syndrom durch die Trias aus Hämolyse (LDH > 600 U / L, Haptoglobin erniedrigt), erhöhten Leberenzymen (GOT > $70 \mathrm{U} / \mathrm{L}$ ) und Thrombozytopenie (Thrombozyten < $100000 / \mu \mathrm{l}$ ) [2]. Zudem können unspezifische Symptome wie allgemeines Unwohlsein, (rechtsseitige) Oberbauchschmerzen, Übelkeit und Erbrechen, Kopfschmerzen und Sehstörungen auftreten. In 82-88\% der Fälle besteht eine Hypertonie und in $86-100 \%$ der Fälle eine Proteinurie [3].
Im Hinblick auf die mütterliche und kindliche Morbidität und Mortalität ist es vor allem die frühe Form des HELLPSyndroms (Auftreten vor der vollendeten 34. SSW), die in der industrialisierten Welt von großer Relevanz ist. Aber auch die Late-Onset-Form dieser Erkrankung gehört global betrachtet zu den Hauptgründen der mütterlichen und kindlichen peripartalen Todesfälle. Die derzeit einzig bekannte kurative Therapie ist die Entbindung. Jedoch sollte bei Auftreten einer frühen Präeklampsie die Prolongation der Schwangerschaft und Induktion der fetalen Lungenreife mittels Kortikosteroiden erwogen werden [4]. Ziel ist eine Verlängerung der Schwangerschaft über die abgeschlossene 30. SSW hinaus, durch engmaschige Kontrollen der Laborparameter und eine individualisierte antihypertensive sowie antikonvulsive Therapie.

\section{Besonderheiten beim Very early HELLP-Syndrom}

Ganz anders sieht es jedoch bei Auftreten eines sehr frühen HELLP-Syndroms (Very early HELLP-Syndrom) aus. Hier ist im Hinblick auf die erheblichen maternalen Risiken und die sehr fragwürdige Prognose des Kindes bei einer Entbindung vor oder an der Grenze der extrauterinen Überlebensfähigkeit $(\leq 24$. SSW) unter maximaler intensivmedizinischer Intervention insbesondere auch ein Schwangerschaftsabbruch zu diskutieren. Das Ansprechen dieses Entscheidungsweges löst bei den betroffenen Paaren nicht selten eine existenzielle emotionale Krise aus, die mit einem nachhaltigen Hinterfragen aller bisher getroffenen Entscheidungen verbunden ist. Sehr schnell kommt es zu Vertrauensbrüchen, insbesondere, wenn es in der professionellen Beratung nicht gelingt, einen weitgehend nondirektiven und von den Eltern mitbestimmten Weg einzuschlagen. 
Merke

Beim Very early HELLP-Syndrom ist durch die maternale Gefährdung ein Zeitdruck gegeben. Deshalb erfordert die Beratung des Elternpaares viel

Fingerspitzengefühl und eine gute Einschätzung des individuellen Krankheitsverlaufes.

\section{Beratung der Eltern}

Es gilt schon in den ersten Gesprächen mit den Eltern, klar zu machen, dass die Diagnose eines extrem frühen HELLP-Syndroms äußerst selten ist und meist ohne wesentlichen zeitlichen Vorlauf gestellt wird [2]. Dies ist ebenso wichtig wie eine Relativierung subjektiv empfundener Verhaltensfehler der Schwangeren.

Die Beantwortung der Frage nach persönlichen und durch das professionelle Umfeld zu verantwortenden Versäumnissen nimmt nicht selten einigen Gesprächsraum ein.

Darüber hinaus müssen die Ergebnisse der Sonografie (Doppler, Fruchtwassermenge, Biometrie, Zeichen einer intrauterinen Mangelversorgung etc.) erläutert und der Interpretationsspielraum bezüglich der Bedeutung für das „Outcome“ des Kindes veranschaulicht werden. Ob neben den sonografischen und charakteristischen maternalen Befunden zur Prädiktion und Diagnostik einer frühen Präeklampsie auch der sFlt-1/PIGF-Quotient herangezogen werden kann, dessen Anwendung auf der Basis der Empfehlungen einer internationalen Konsensusgruppe bei Einlingsschwangerschaften ab der abgeschlossenen 24. SSW beruht, bleibt abzuwarten. Für ein sehr frühes HELLP-Syndrom ist dieser Quotient nicht validiert, kann bei der Diagnose und insbesondere in der Diskussion des zu erwartenden Verlaufes dennoch eine Hilfe sein.

\section{Fallbeispiele}

In dieser Arbeit sollen 2 exemplarische Fallbeispiele mit Auftreten eines sehr frühen HELLP-Syndroms vor der 24. SSW und das Management in diesen Situationen vorgestellt und diskutiert werden.

\section{Fall 1 - Erstgravida (29) in der $18+3$ SSW}

Die stationäre Aufnahme der 29-jährigen Erstgravida erfolgte in der $18+3$ SSW mit seit 2 Wochen bestehenden Oberbauchschmerzen und einem Blutdruck von 155 / 102 mmHg. Nebenbefundlich wurde eine Hypothyreose diagnostiziert. Sonografisch zeigte sich eine Plazentainsuffizienz mit schwerer symmetrischer fetaler Wachstumsretardierung und Oligohydramnion. Die fetalen Doppler waren unauffällig, die uterinen Doppler jedoch beidseits erhöht und zeigten ein Notching. Des Weiteren fiel bei der sonografischen Untersuchung des Feten ein hyperechogener Darm auf. Darüber hinaus bestand kein Anhalt für fetale Fehlbildungen. Die maternale Thrombozytenzahl lag mit $239000 / \mu$ im Normbereich, die GOT war mit 246 U/I erhöht. Das $\beta$-HCG lag mit $65000 \mathrm{IE} / \mathrm{I}$ etwas über dem für diese Schwangerschaftswoche zu erwartenden Normwert (6 Tage später mit 40000 IE / I im Normbereich). Der sFlt-1 / PIGF-Quotient war mit 310 deutlich erhöht. Bei persistierenden starken Schmerzen im Magenbereich erfolgte ein chirurgisches und internistisches Konsil, jedoch ohne Anhalt für eine akute internistische oder chirurgische Ursache der Beschwerden.

Bei Verdacht auf eine frühe, schwere Präeklampsie wurde die Schwangere werteneutral und umfassend beraten. Sie entschied sich zunächst gegen einen Schwangerschaftsabbruch und für einen Prolongationsversuch. Die Blutdruckeinstellung erfolgte mit Methyldopa und eine Flüssigkeitsbilanzierung wurde durchgeführt. Zudem erhielt die Frau Magnesiumsulfat i. v. und im Verlauf, bei sinkenden Thrombozytenzahlen, eine Kortisonstoßtherapie mit jeweils $32 \mathrm{mg}$ Methylprednisolon an 2 aufeinander folgenden Tagen. Bei erhöhten Infektparametern erfolgte eine antibiotische Therapie. Weitere durchgeführte Untersuchungen wie eine initiale CVS / AC, TORCH-Untersuchung, Hepatitisserologie, HITDiagnostik und Eiweißausscheidung im Urin zeigten unauffällige Ergebnisse. Ein hämolytisch-urämisches Syndrom (HUS) wurde ausgeschlossen.

Bei im Verlauf zunehmender Beschwerdesymptomatik (Prodrome) der Patientin und pathologischen fetalen Dopplern im Sinne einer Zentralisation wurde-nach mehrfachen ausführlichen Beratungen der Frau - in der $19+3$ SSW die Indikation zum Schwangerschaftsabbruch gestellt und durch eine Einleitung der Geburt mittels Misoprostol vaginal (nach entsprechender Aufklärung über den Off-Label-Use) durchgeführt. Die Frau erhielt eine psychologische Betreuung.

Die Laborparameter stabilisierten sich nach der Entbindung rasch wieder. Der Patientin ging es binnen weniger Tage klinisch deutlich besser und daher konnte die stationäre Behandlung bereits am 5. Tag nach Abbruch der Schwangerschaft beendet werden. Die Obduktion zeigte einen hypotrophen weiblichen Feten ohne Fehlbildungen. Die Plazenta war mit $83 \mathrm{~g}$ untergewichtig und zeigte Zottenreifungsstörungen sowie disseminierte alte Infarkte und ältere Blutungen. Der humangenetische Befund war unauffällig.

Zwei Monate postpartal war die klinische Symptomatik weitestgehend verschwunden, die Laborparameter befanden sich im Normbereich. Es bestand jedoch weiterhin eine arterielle Hypertonie, welche bereits präexistent gewesen sein könnte. Bei wiederholt nachweisbaren Antiphospholipid-Antikörpern wurde nach Abbruch der Schwangerschaft die Verdachtsdiagnose eines 
Antiphospholipid-Syndroms gestellt und die Beratung bezüglich einer weiteren Schwangerschaft in dieser Richtung ausgestaltet.

\section{Fall 2 - Erstgravida (32) in $16+2$ SSW}

Die Aufnahme der 32-jährigen Erstgravida erfolgte in der $16+2$ SSW mit epigastrischen Beschwerden. Nebenbefundlich waren eine Hypothyreose und Hüftdysplasie beidseits bekannt. Die Schwangerschaft war im Rahmen einer Kinderwunschbehandlung durch Stimulation entstanden. Die Thrombozytenzahl lag bei Aufnahme bei $66000 / \mu \mathrm{l}$, die GOT bei $136 \mathrm{U} / \mathrm{I}$ und das $\beta$-HCG bei 61000 IE/l. Der sFlt-1/PIGF-Quotient war mit > 1000 stark erhöht. Eine Hypertonie bestand initial nicht. Sonografisch zeigte sich eine asymmetrische fetale Wachstumsrestriktion ohne Anhalt für Fehlbildungen oder eine Molenschwangerschaft. Die maternale Doppler-Untersuchung der Aa. uterinae zeigte einen auffälligen Befund beidseits mit erhöhtem Widerstand und Notching. Weitere durchgeführte Untersuchungen wie eine TORCHUntersuchung, Hepatitisserologie und Kontrolle der Eiweißausscheidung im Urin zeigten unauffällige Ergebnisse. Ein hämolytisch-urämisches Syndrom (HUS) wurde ausgeschlossen. Es wurde ein gastroenterologisches und kardiologisches Konsil durchgeführt, welches sich mit Ausnahme eines Schleimhauterythems im Antrum unauffällig zeigte.

Bei Verdacht auf ein Very early HELLP-Syndrom erfolgte die Therapie mit Magnesiumsulfat i.v. und die mehrfache Gabe von Methylprednisolon (initial $64 \mathrm{mg}$ bei Aufnahme sowie erneut $64 \mathrm{mg}$ am Folgetag und $32 \mathrm{mg}$ bzw. $16 \mathrm{mg}$ an den beiden darauffolgenden Tagen). Zunächst gelang es, die Thrombozytenzahl zu stabilisieren, die Leberwerte fielen und der Schwangeren ging es unter analgetischer Therapie klinisch deutlich besser. In dieser Zeit fanden mehrfache Gespräche bezüglich der Prolongation oder des Abbruchs der Schwangerschaft statt. Die Patientin war über lange Zeit bezüglich einer Entscheidung sehr ambivalent.

Am 5. Tag nach Aufnahme kam es klinisch und laborchemisch zu einer erneuten Progredienz des Very early HELLP-Syndroms. Es zeigte sich eine milde Hypertonie. Erschwerend kam hinzu, dass die Religionszugehörigkeit der Frau eine Bluttransfusion nicht erlaubte. Dies war im Hinblick auf eine massive Hämolyse und fallende Thrombozytenzahlen bei der weiteren Behandlungsplanung zu bedenken. Schließlich wurde im Einvernehmen mit der Patientin in der $17+0$ SSW die Indikation zum Schwangerschaftsabbruch gestellt. Der Schwangerschaftsabbruch erfolgte durch eine Einleitung der Geburt mittels Mifepriston und Misoprostol. Zur Stabilisierung der Thrombozytenzahl erhielt die Frau erneut initial $64 \mathrm{mg}$ Methylprednisolon i. v., sowie $32 \mathrm{mg}$ bzw. $16 \mathrm{mg}$ an den beiden darauffolgenden Tagen.
Nach der Geburt eines avitalen Mädchens erfolgte die instrumentelle Nachtastung sowie bei verstärkter Blutung die Gabe von Nalador und Einlage einer intrakavitären Tamponade. Bei einem Hämoglobinwert von $8,8 \mathrm{~g} / \mathrm{dl}$ erhielt die Patientin eine Eiseninfusion. Im weiteren Verlauf stabilisierten sich die Laborwerte und es kam zu einer deutlichen Besserung der klinischen Symptomatik, sodass die Frau eine Woche nach Abbruch der Schwangerschaft in die ambulante Betreuung entlassen werden konnte.

Die Obduktion zeigte einen hypotrophen Feten (Gewicht entsprechend der 12. SSW) mit geringgradiger Retrognathie und tiefsitzenden Ohren. Die Plazenta war mit $44 \mathrm{~g}$ deutlich untergewichtig. Es fielen dissoziierte Zottenreifungsstörungen, ausgeprägte Stromablutungen und fokale Zottennekrosen auf. Die humangenetische Untersuchung lieferte eine unauffällige Karyotypisierung. Das Thrombophiliescreening zeigte einen unauffälligen Befund. Einen Monat später erfolgte die Wiedervorstellung der Patientin. Ihr ging es klinisch gut und sie erkundigte sich über Risiken und Präventionsmöglichkeiten im Falle einer erneuten Schwangerschaft. Die Beratung bezüglich einer weiteren Schwangerschaft erfolgte auf der Basis der erhobenen Befunde und der aktuellen Literatur. 
Ein Jahr später kam es zu einem Abort in der 9. SSW ohne Komplikationen oder verstärkte Blutungen. Im Jahr darauf folgte eine erfolgreiche Schwangerschaft, die erfolgreich ausgetragen werden konnte. Es wurde eine intensive Schwangerschaftsbetreuung gewährleistet und es kam zu keinerlei Zeichen einer Präeklampsie.

\section{Diskussion}

Ein HELLP-Syndrom wird in 0,5-0,9\% aller Schwangerschaften und in 10-20\% aller Schwangerschaften mit schwerer Präeklampsie manifest [2][3]. 10\% aller HELLPSyndrome beginnen vor der 27. SSW [4].

Das Auftreten eines HELLP-Syndroms vor der extrauterinen Lebensfähigkeit des Feten (Very early HELLP-Syndrom) ist äußerst selten. Der sehr frühe Beginn der Erkrankung steht nicht selten im Zusammenhang mit weiteren Komorbiditäten, z. B. einer fetalen Triploidie, einer Partialmole oder einem Antiphospholipid-Syndrom [5][6][7][8]. Dies muss daher in der klinischen Diagnostik Berücksichtigung finden (Infobox).

Als weitere Risikofaktoren für ein HELLP-Syndrom gelten Multiparität, erhöhtes mütterliches Alter, afroamerikanische Abstammung, das Auftreten eines HELLP-Syndroms in der Eigen- oder Familienanamnese oder vorbestehende rheumatische Erkrankungen (z.B. systemischer Lupus erythematodes) [9][10][11][12][13]. Demgegenüber sind in der Literatur Fälle beschrieben, die keinen dieser Risikofaktoren erkennen lassen [14].

Die Präeklampsie und die spezielle Form des Very early HELLP-Syndroms sind plazentaassoziierte Schwangerschaftserkrankungen. Bei ihrer Pathogenese-die bisher nicht vollständig verstanden wurde - spielen immunologische und genetische Faktoren eine Rolle [15][16][17][18]. Dabei werden eine Kombination mehrerer Genvarianten und Störungen in der Stoffwechselkaskade mit weiteren maternalen und Umweltfaktoren bei der Ätiologie dieser Erkrankung diskutiert [19][20]. Eine immunologische Anpassungsstörung führt vermutlich im 1 . Trimester zu einer maternalen Abstoßungsreaktion gegenüber den sich einnistenden Trophoblasten [21]. Es werden antiangiogenetische Faktoren (z.B. soluble fms-like Tyrosinkinase$1=$ sFlt-1) ausgeschüttet, die zu einer Entzündungsreaktion und Aktivierung der Gerinnungskaskade mit thrombotischer Mikroangiopathie führen [15][20]. Ein Teil der Pathophysiologie und der maternalen Symptome lässt sich so schlüssig erklären, ein Teil jedoch nicht.

INFO

Differenzialdiagnosen des HELLP-Syndroms (nach

Daten aus [22])

Schwangerschaftsassoziierte Erkrankungen:

- schwangerschaftsassoziierte Thrombozytopenie
- akute Schwangerschaftsfettleber

Infektionserkrankungen, Entzündungen

- Virushepatitis

- Cholezystitis bzw. Entzündung der Gallenwege

- Entzündung der oberen Harnwege

- Gastritis

- Magenulkus

- akute Pankreatitis

Sonstige Thrombozytopenien

- idiopathische Thrombozytopenie (ITP)

- systemischer Lupus erythematodes (SLE)

- Antiphospholipid-Syndrom (APS)

thrombotische Mikroangiopathien

- thrombotisch-thrombozytopenische Purpura (TTP)

- hämolytisch-urämisches Syndrom (HUS)

\section{Klinische Betreuung}

Die klinische Betreuung des Very early HELLP-Syndroms hält viele Stolpersteine bereit. Beispielhaft kam es in beiden Fallberichten zu einem akuten Auftreten der Erkrankung ohne wesentliche Vorwarnzeichen. Entsprechend musste ein für die betroffenen Paare tragfester Entscheidungsweg stringent angebahnt werden. Neben dem Ausschluss von Differenzialdiagnosen (siehe Infobox) ist ein wesentliches Element die „Entschleunigung“ des Krankheitsgeschehens, indem die Symptome der Erkrankung und die Krankheitsentwicklung medikamentös beeinflusst werden-also eine maternale Stabilisierung angestrebt wird.

In beiden Fällen mussten die Schwangerschaften bei erneutem Progress der Erkrankung aus maternaler Indikation und im Hinblick auf die infauste kindliche Prognose beendet werden. Hervorzuheben ist, dass sich jeweils ein deutlich erhöhter sFlt-1/PIGF-Quotient zeigte. Dieser ist zwar erst für eine spätere Schwangerschaftswoche validiert [23]. Er kann jedoch gerade in diesen frühen Schwangerschaftswochen als Parameter herangezogen werden, der die Diagnose eines Very early HELLP-Syndroms stützt und zusammen mit den weiteren klinischen und laborchemischen Parametern die Dringlichkeit zu handeln unterstreicht, da der Quotient bei der frühen Präeklampsie und dem frühen HELLP-Syndrom stärker ansteigt als bei den späten Formen [16]. Für eine valide Aussage sind hier jedoch die Ergebnisse weiterer klinischer Studien abzuwarten.

Ein individuelles und mit den Wünschen der Schwangeren abgestimmtes Vorgehen scheint in vergleichbaren Situationen mehr als ratsam. Dabei ist Voraussetzung, dass die Frau zu jedem Zeitpunkt über den Schweregrad der Erkrankung sowie die damit verbundenen Komplikationen und eine möglicherweise bevorstehende Beendigung der Schwangerschaft informiert ist. Dem Vorgehen 
stimmt sie in der Situation angepassten Beratungen im Sinne eines „Informed Consent“ zu [24][25].

Es hat sich bewährt, dass die betroffenen Paare die Informationen nicht nur durch den Geburtshelfer bzw. Pränataldiagnostiker erhalten, sondern auch durch Hebammen, Kinderärzte, Anästhesisten, Psychologen und ggf. Seelsorger beraten werden. Es wird für sie spürbar, dass man versucht, sich dem Problem von mehreren Seiten (mütterlich, kindlich, intensivmedizinisch, psychologisch, seelsorgerisch...) zu nähern und dabei die individuellen Belange von Mutter, Vater und Kind zu berücksichtigen.

\section{Merke}

Über den gesamten Behandlungszeitraum sollte eine intensive maternale Überwachung gewährleistet sein. Trotz des Zeitdrucks, für die Mutter vermeidbare Komplikationen auszuschließen, darf nie der Eindruck entstehen, das Kind in utero vorschnell aufgegeben zu haben. Es geht nicht ausschließlich darum, ob eine Prolongation der Schwangerschaft medizinisch vertretbar ist, sondern auch darum, den Eltern Zeit zu verschaffen, sich auf den Gedanken eines Schwangerschaftsabbruchs einzulassen. Das heißt aber auch, die Option einer aktiven Schwangerschaftsbeendigung nicht generell auszuschließen.

In beiden vorgestellten Fällen wurde der Versuch unternommen, den Verlauf des Very early HELLP-Syndroms also die Dysbalance antiangiogenetischer und entzündlicher Faktoren - mittels Kortikosteroiden zu beeinflussen [26]. Eine Hochdosistherapie mit Kortikosteroiden wird in der Literatur nicht ohne Grund kontrovers diskutiert. Einige Studien zeigen verbesserte Laborergebnisse und eine Reduktion der maternalen Morbidität durch eine frühe Therapie mit Kortikosteroiden [11][12] sowie nachweisbar reduzierte Entzündungsmediatoren (wie z. B. sFlt1) im maternalen Blut [26][27][28]. Andere fanden in ihrer Studie jedoch keine Verbesserung des maternalen Outcomes und keinen Unterschied im Hinblick auf schwerwiegende Komplikationen [29]. Zu diesen zählen beispielsweise die vorzeitige Plazentalösung, das akute Nierenversagen, das Lungenödem, die disseminierte intravasale Koagulopathie sowie die perinatale mütterliche und kindliche Mortalität. In Kenntnis dieser Ergebnisse sollte im Falle einer maternalen Befundverschlechterung der Symptome eine zeitnahe Entbindung angestrebt werden [22]. Diese Entscheidung bedeutet nicht selten eine klinische und ethische Gratwanderung. Soweit möglich wird im Rahmen des Schwangerschaftsabbruchs eine vaginale Entbindung angestrebt. Je nach klinischem Verlauf kann jedoch auch eine Sectio parva erforderlich werden. 


\section{Maternale und kindliche Risiken einer Folgeschwangerschaft}

Bei erneutem Kinderwunsch der Frau stellt sich die Frage bezüglich des Wiederholungsrisikos eines Very early HELLP-Syndroms und einer möglichen Früherkennung bzw. Prävention. In der Schwangerschaft zuvor erhobene Befunde können dabei wegweisend sein, wenngleich eine genaue individuelle Risikoeinschätzung derzeit nicht möglich ist. Generell wird das Wiederholungsrisiko eines HELLP-Syndroms in einer Folgeschwangerschaft mit 19$27 \%$ angegeben, das Auftreten einer Präeklampsie nach HELLP-Syndrom in einer vorigen Schwangerschaft mit $43 \%$ [30]. Für das Wiederholungsrisiko eines Very early HELLP-Syndroms gibt es keine verlässlichen Zahlen. Es ist jedoch - ableitbar aus den Untersuchungen zur Wiederholungswahrscheinlichkeit eines frühen HELLP-Syndroms - als hoch einzuschätzen.

Mittelpunkt der Beratung müssen neben den maternalen Risiken auch die vom Gestationsalter abhängigen kindlichen Risiken sein. So zeigen Frühgeborene, die im Rahmen einer frühen Präeklampsie geboren werden, gegenüber Frühgeborenen gleichen Gestationsalters ohne Präeklampsie der Mutter eine höhere perinatale Mortalität (13 vs. $7 \%, p=0,03$ ) und Säuglingssterblichkeit ( 16 vs. $9 \%, p=0,03$ ), sowie ein $20 \%$ niedrigeres Geburtsgewicht (1150 vs. 1430 g, p<0,001). Die Frühgeborenen waren häufiger SGA-Kinder (22 vs. $9 \%$, p < 0,001 ) und hatten mehr neonatale Komplikationen [31]. Es ist daher sehr wichtig, in der aktuellen und in einer Folgeschwangerschaft, das fetale Wachstum ab der 18. SSW zu beobachten, um eine frühe Wachstumsrestriktion mit in die weiteren Entscheidungen (Fortsetzung der Schwangerschaft oder Schwangerschaftsabbruch) einzubeziehen, da die oben genannten Komplikationen vor allem mit SGA-Kindern assoziiert sind [31]. Mittels Screeningalgorithmen im 1. Trimenon (mütterliche Anamnese, biophysikalische und biochemische Marker) können zwar bei einer Falschpositivrate von $10 \%$ ca. $96 \%$ der Schwangerschaften identifiziert werden, die aufgrund einer Präeklampsie vor der 34.SSW entbunden werden, allerdings hilft dies in der Entscheidung und im Management bezüglich einer weiteren Schwangerschaft nicht wirklich weiter, denn alleine der Umstand, dass die Frau bereits ein Very early HELLP-Syndrom in der Eigenanamnese aufweist, bedeutet für sie ein deutlich erhöhtes Wiederholungsrisiko für eine Frühgeburt [32].

Eine in den Niederlanden durchgeführte retrospektive Analyse an 120 Frauen nach einer 1 . Schwangerschaft mit einer frühen Präeklampsie konnte einen in der Mehrzahl günstigen Verlauf in der folgenden Schwangerschaft zeigen [33]. Dies wird durch weitere aktuelle Daten gestützt, die Anlass geben, darüber nachzudenken, dass in der Vergangenheit das Risiko für das erneute Auftreten einer Präeklampsie in der Folgeschwangerschaft und das damit verbundene schlechte Outcome von Mutter und Kind zu negativ bewertet wurden [34]. Da jedoch die beschriebenen Fallzahlen von Very early HELLP-Syndromen sehr niedrig sind, sind alle diese Aussagen sehr vorsichtig zu bewerten. Eigene Erfahrungen in unserem Zentrum geben Anlass - mit Blick auf die aktuelle Literatur -, betroffenen Paaren Mut für eine weitere Schwangerschaft zu machen.

\section{Betreuung bei erneuter Schwangerschaft}

Merke
Im Falle einer erneuten Schwangerschaft sollte bei
einem erhöhten Risiko für ein erneutes Auftreten eines
schweren HELLP-Syndroms oder einer Präeklampsie
eine engmaschige Betreuung der Frau - möglichst in
Anbindung an ein Perinatalzentrum - erfolgen, um
eine umfassende Betreuung von Mutter und Kind bei
Komplikationen zu gewährleisten [35].

Neben dem erweiterten 1. Trimesterscreening sollten ab der 18. SSW 2-wöchentliche ärztliche Kontrollen erfolgen-idealerweise im Wechsel zwischen dem betreuenden Frauenarzt und dem Perinatalzentrum. Zur Überwachung der intrauterinen Entwicklung des Feten dienen engmaschige Doppler- und Wachstumskontrollen. Inwieweit die serielle Bestimmung des sFlt-1 / PIGF-Quotienten bei der Überwachung hilfreich sein kann, ist derzeit noch unklar. Es fehlen Daten, dass die konsequente serielle Nutzung des Quotienten das Outcome von Mutter und Kind in einer Schwangerschaft nach einem Very early HELLP-Syndrom verbessert. In jedem Fall kann die Bestimmung des sFlt-1/PIGF Quotienten in Grenzsituationen helfen zu differenzieren, in welche Richtung sich der weitere Schwangerschaftsverlauf-unter dem hohen Risiko, schon einmal ein Very early HELLP-Syndrom in der Eigenanamnese erlebt zu haben - gestalten wird, um den besten Entbindungszeitpunkt festzulegen [36].

Eine in der Frühschwangerschaft begonnene Einnahme von niedrig dosiertem ASS (150 mg/d vor der 16 + 0 SSW begonnen) zeigt eine signifikante Risikoreduktion für das erneute Auftreten einer frühen Präeklampsie und sollte daher im Zustand nach einem Very early HELLP-Syndrom unbedingt empfohlen werden [37]. Die derzeitige Datenlage legt nahe, die Substitution von Kalzium und Vitamin D ebenso zu empfehlen [38][39][40]. Allerdings fehlen auch hier für eine umfassende Bewertung ausreichende Studiendaten. Dies gilt in besonderer Weise für andere diskutierte präventive Maßnahmen, wie beispielsweise eine salzreiche Diät, und andere. Neben dem erhöhten Risiko für das Auftreten einer erneuten Präeklampsie bzw. eines erneuten Very early HELLP-Syndroms muss in der 
Beratung auch das erhöhte Risiko bezüglich des Auftretens kardiovaskulärer Erkrankungen oder Depressionen unabhängig von einer angestrebten weiteren Schwangerschaft thematisiert werden [41].

Postpartale Erfahrungen mit der Plasmapherese geben Anlass, auch über einen Einsatz derselben in der Schwangerschaft nachzudenken [42][43]. Ziel wäre das Erreichen der fetalen Lebensfähigkeit außerhalb des Mutterleibes bzw. eine Prolongation der Schwangerschaft in ein höheres Gestationsalter. In einer Pilotstudie wurden 3 Frauen ab der 28. SSW mittels Plasmapherese von sFlt-1 behandelt. Es gelang eine Prolongation der Schwangerschaften durch Kontrolle der maternalen Symptome über 15-23 Tage [44]. Ein solches Vorgehen ist jedoch weiterhin experimentell und sollte ausschließlich in Studien durchgeführt werden. Bei einem Very early HELLP-Syndrom ist zudem die erforderliche Zeitspanne für den Feten bis zum Erreichen der Lebensfähigkeit außerhalb des Mutterleibes zu hinterfragen, die unter Umständen 4-6 Wochen betragen kann. Es kann nicht Ziel eines sinnvollen geburtshilflichen Managements sein-sollte eine Prolongation gelingen -, eine Entbindung in der 23.-24. Schwangerschaftswoche bei ausgeprägtem SGA-Wachstum unter katastrophalen fetalen Bedingungen anzustreben. Von Interesse ist darüber hinaus die Anwendung monoklonaler Antikörper. Eculizumab beispielsweise ist zugelassen zur Behandlung des atypischen hämolytisch-urämischen Syndroms (aHUS) und führt zu einer Hemmung oben angedeuteter immunologischer Reaktionen. Unter dieser Medikation ist bereits eine Prolongation der Schwangerschaft über 17 Tage in der 26. SSW beschrieben worden [45]. Darüber hinaus gibt es mehrere Fallberichte zur Therapie des aHUS in der Frühschwangerschaft [46][47][48]. Eculizumab hat jedoch bei der Therapie des Very early HELLP-Syndroms keine Zulassung. Noch reichen die Daten klinischer Studien aus, um eine Anwendung generell zu rechtfertigen. Die Substanz ist jedoch gerade bei dieser Fragestellung interessant und sollte in weiteren Studien untersucht werden.

FAZIT

Das Very early HELLP-Syndrom stellt eine große Herausforderung für alle Beteiligten dar. Es gilt, dieser interdisziplinär und mit einer klaren Strategie zu begegnen. Das Ziel ist, alle Entscheidungswege für die betroffenen Paare nachvollziehbar darzustellen und die Untersuchungsergebnisse sowie deren Veränderungen weitreichend zu erläutern. Dabei ist es von entscheidender Bedeutung, Zeit zu gewinnen, um tragfeste Entscheidungen anzubahnen, ohne eine massive maternale Gefährdung in Kauf zu nehmen. Dabei ist von allen beteiligten Fachkräften viel fachliches Augenmaß, aber auch eine hohe menschliche Kompetenz zu fordern. 


\section{Über diesen Artikel}

Dieser Artikel ist beruht auf der Originalveröffentlichung der Autoren: Pauluschke-Fröhlich J, Kagan K, Bihler M et al. Very early HELLP-Syndrom - vergeblicher Aktionismus oder geordneter Rückzug?. Geburtshilfe und Frauenheilkunde 2018; 78(07): 671-676. doi:10.1055/a-0589-4227

\section{Autorinnen / Autoren}

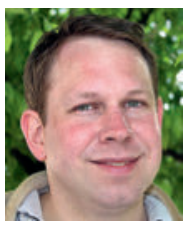

Dr. med. Jan Pauluschke-Fröhlich ist Oberarzt Geburtshilfe, Department für Frauengesundheit am Universitätsklinikum Tübingen.

Prof. Dr. med. Karl-Oliver Kagan ist Leitender Oberarzt Pränataldiagnostik DEGUM III, Department für Frauengesundheit am Universitätsklinikum Tübingen.

Dr. med. Manuela Bihler ist Fachärztin, Frauenklinik, Klinikum am Steinenberg Reutlingen.

Prof. Dr. med. Harald Abele ist Leiter des Studiengangs Hebammenwissenschaft und stellvertretender Ärztlicher Direktor Geburtshilfe, Department für Frauengesundheit am Universitätsklinikum Tübingen.

\section{Korrespondenzadresse}

Dr. med. Jan Pauluschke-Fröhlich

Geburtshilfe I Department für Frauengesundheit

Universitätsklinikum Tübingen

Calwerstraße 7

72076 Tübingen

E-Mail: pauluschke@gmx.net

Literatur

[1] Weinstein L. Syndrome of hemolysis, elevated liver enzymes, and low platelet count: a severe consequence of hypertension in pregnancy. Am J Obstet Gynecol 1982; 142: 159-167

[2] Geary M. The HELLP syndrome. Br J Obstet Gynaecol 1997; 104: 887-891

[3] Karumanchi SA, Maynard SE, Stillman IE. et al. Preeclampsia: a renal perspective. Kidney Int 2005; 67: 2101-2113

[4] Magann EF, Martin jr. JN. Twelve steps to optimal management of HELLP syndrome. Clin Obstet Gynecol 1999; 42: 532-550

[5] Broekhuizen FF, Elejalde R, Hamilton PR. Early-onset preeclampsia, triploidy and fetal hydrops. J Reprod Med 1983; 28: 223-226

[6] Alsulyman OM, Castro MA, Zuckerman E. et al. Preeclampsia and liver infarction in early pregnancy associated with the antiphospholipid syndrome. Obstet Gynecol 1996; 88 (4 Pt 2): 644-646

[7] Sherer DM, Dalloul M, Stimphil R. et al. Acute onset of severe hemolysis, elevated liver enzymes, and low platelet count syndrome in a patient with a partial hydatidiform mole at 17 weeks gestation. Am J Perinatol 2006; 23: 163-166
[8] Tsirigotis P, Mantzios G, Pappa V. et al. Antiphospholipid syndrome: a predisposing factor for early onset HELLP syndrome. Rheumatol Int 2007; 28: 171-174

[9] Audibert F, Friedman SA, Frangieh AY. et al. Clinical utility of strict diagnostic criteria for the HELLP (hemolysis, elevated liver enzymes, and low platelets) syndrome. Am J Obstet Gynecol 1996; 175: 460-464

[10] Barton JR, Sibai BM. Diagnosis and management of hemolysis, elevated liver enzymes, and low platelets syndrome. Clin Perinatol 2004; 31: 807-833 vii

[11] Padden MO. HELLP syndrome: recognition and perinatal management. Am Fam Physician 1999; 60: 829-836 839

[12] Sibai BM. Diagnosis and management of gestational hypertension and preeclampsia. Obstet Gynecol 2003; 102: 181-192

[13] Sibai BM. Imitators of severe preeclampsia. Obstet Gynecol 2007; 109: 956-966

[14] Berry EL, lqbal SN. HELLP syndrome at 17 weeks gestation: a rare and catastrophic phenomenon. J Clin Gynecol Obstet 2014; 3: 147-150

[15] Abildgaard U, Heimdal K. Pathogenesis of the syndrome of hemolysis, elevated liver enzymes, and low platelet count (HELLP): a review. Eur J Obstet Gynecol Reprod Biol 2013; 166: $117-123$

[16] Schaarschmidt W, Rana S, Stepan $\mathrm{H}$. The course of angiogenic factors in early- vs. late-onset preeclampsia and HELLP syndrome. J Perinat Med 2013; 41: 511-516

[17] Várkonyi T, Nagy B, Füle T. et al. Microarray profiling reveals that placental transcriptomes of early-onset HELLP syndrome and preeclampsia are similar. Placenta 2011; 32 (Suppl.) S21-S29

[18] Haram K, Mortensen JH, Nagy B. Genetic aspects of preeclampsia and the HELLP syndrome. J Pregnancy 2014; 2014: 910751

[19] Nelson J, Lewis B, Walters B. The HELLP syndrome associated wiht fetal medium-chain acyl-CoA dehydrogenase deficiency. J Inherit Metab Dis 2000; 23: 518-519

[20] Fang C], Richards A, Liszewski MK. et al. Advances in understanding of pathogenesis of aHUS and HELLP. Br J Haematol 2008; 143: 336-348

[21] Mihu D, Costin N, Mihu CM. et al. HELLP syndrome - a multisystemic disorder. J Gastrointestin Liver Dis 2007; 16: 419424

[22] Haram K, Svendsen E, Abildgaard U. The HELLP syndrome: clinical issues and management. A Review. BMC Pregnancy Childbirth 2009; 9: 8

[23] Verlohren S, Herraiz I, Lapaire O. et al. The sFlt-1 / PIGF ratio in different types of hypertensive pregnancy disorders and its prognostic potential in preeclamptic patients. Am J Obstet Gynecol 2012; 206: 58.e1-58.e8

[24] Ahmed A. Informed decision making and abortion: crisis pregnancy centers, informed consent, and the first amendment. J Law Med Ethics 2015; 43: 51-58

[25] Glantz LH. Pregnancy and informed consent to research. J Am Med Womens Assoc(1972) 2000; 55: 263-264

[26] Wallace K, Martin jr.jN, Tam Tam K. et al. Seeking the mechanism(s) of action for corticosteroids in HELLP syndrome: SMASH study. Am J Obstet Gynecol 2013; 208: 380.e1-380.e8

[27] Martin jr.JN, Rose $\mathrm{CH}$, Briery CM. Understanding and managing HELLP syndrome: the integral role of aggressive glucocorticoids for mother and child. Am J Obstet Gynecol 2006; 195: 914-934 
[28] Martin jr.JN, Thigpen BD, Rose CH. et al. Maternal benefit of high-dose intravenous corticosteroid therapy for HELLP syndrome. Am J Obstet Gynecol 2003; 189: 830-834

[29] Fonseca JE, Méndez F, Cataño C. et al. Dexamethasone treatment does not improve the outcome of women with HELLP syndrome: a double-blind, placebo-controlled, randomized clinical trial. Am J Obstet Gynecol 2005; 193: 1591-1598

[30] Sullivan CA, Magann EF, Perry jr. KG. et al. The recurrence risk of the syndrome of hemolysis, elevated liver enzymes, and low platelets (HELLP) in subsequent gestations. Am J Obstet Gynecol 1994; 171: 940-943

[31] van Esch JJA, van Heijst AF, de Haan AF]. et al. Early-onset preeclampsia is associated with perinatal mortality and severe neonatal morbidity. J Matern Fetal Neonatal Med 2017; 30: 2789-2794

[32] Akolekar R, Syngelaki A, Poon L. et al. Competing risks model in early screening for preeclampsia by biophysical and biochemical markers. Fetal Diagn Ther 2013; 33: 8-15

[33] van Rijn BB, Hoeks LB, Bots ML. et al. Outcomes of subsequent pregnancy after first pregnancy with early-onset preeclampsia. Am J Obstet Gynecol 2006; 195: 723-728

[34] Seeho SK, Algert CS, Roberts CL. et al. Early-onset preeclampsia appears to discourage subsequent pregnancy but the risks may be overestimated. Am J Obstet Gynecol 2016; 215: 785. e1-785.e8

[35] Lemonnier M, Beucher G, Morello R. et al. [Subsequent pregnancy outcomes after first pregnancy with severe preeclampsia and delivery before 34 weeks of gestation]. J Gynecol Obstet Biol Reprod (Paris) 2013; 42: 174-183

[36] Álvarez-Fernández I, Prieto B, Rodríguez V. et al. New biomarkers in diagnosis of early onset preeclampsia and imminent delivery prognosis. Clin Chem Lab Med 2014; 52: 1159-1168

[37] Rolnik DL, Wright D, Poon LC. et al. Aspirin versus placebo in pregnancies at high risk for preterm preeclampsia. N Engl ] Med 2017; 377: 613-622

[38] Arain N, Mirza WA, Aslam M. Review-Vitamin D and the prevention of preeclampsia: a systematic review. Pak J Pharm Sci 2015; 28: 1015-1021

[39] DeSousa J, Tong M, Wei J. et al. The anti-inflammatory effect of calcium for preventing endothelial cell activation in preeclampsia. J Hum Hypertens 2016; 30: 303-308
[40] Trumbo PR, Ellwood KC. Supplemental calcium and risk reduction of hypertension, pregnancy-induced hypertension, and preeclampsia: an evidence-based review by the US Food and Drug Administration. Nutr Rev 2007; 65: 78-87

[41] Habli M, Eftekhari N, Wiebracht E. et al. Long-term maternal and subsequent pregnancy outcomes 5 years after hemolysis, elevated liver enzymes, and low platelets (HELLP) syndrome. Am J Obstet Gynecol 2009; 201: 385.e1-385.e5

[42] Eser B, Guven M, Unal A. et al. The role of plasma exchange in HELLP syndrome. Clin Appl Thromb Hemost 2005; 11: 211217

[43] Vafaeimanesh J, Nazari A, Hosseinzadeh F. Plasmapheresis: lifesaving treatment in severe cases of HELLP syndrome. Caspian J Intern Med 2014; 5: 243-247

[44] Thadhani R, Kisner T, Hagmann H. et al. Pilot study of extracorporeal removal of soluble fms-like tyrosine kinase 1 in preeclampsia. Circulation 2011; 124: 940-950

[45] Burwick RM, Feinberg BB. Eculizumab for the treatment of preeclampsia / HELLP syndrome. Placenta 2013; 34: 201-203

[46] Andries G, Karass M, Yandrapalli S. et al. Atypical hemolytic uremic syndrome in first trimester pregnancy successfully treated with eculizumab. Exp Hematol Oncol 2017; 6: 4

[47] Gately R. et al. Life-threatening pregnancy-associated atypical haemolytic uraemic syndrome and its response to eculizumab. Nephrology (Carlton) 2017; 22 (Suppl. 01) 32-35

[48] Servais A, Devillard N, Frémeaux-Bacchi V. et al. Atypical haemolytic uraemic syndrome and pregnancy: outcome with ongoing eculizumab. Nephrol Dial Transplant 2016; 31: 2122-2130

Bibliografie

DOI https://doi.org/10.1055/a-1001-5431

Die Hebamme 2019; 32: 57-65

(c) Georg Thieme Verlag KG Stuttgart · New York ISSN 0932-8122 